\title{
Cyclotron resonance of trilayer graphene
}

\author{
S. H. R. Sena, ${ }^{1,2}$ J. M. Pereira, Jr., ${ }^{2}$ G. A. Farias, ${ }^{2}$ and F. M. Peeters ${ }^{1,2}$ \\ ${ }^{1}$ Department of Physics, University of Antwerp, Groenenborgerlaan 171, B-2020 Antwerpen, Belgium \\ ${ }^{2}$ Departamento de Física, Universidade Federal do Ceará, Fortaleza, Ceará, 60455-760, Brazil
}

(Received 21 April 2012; published 6 August 2012)

\begin{abstract}
The cyclotron resonance energies, the corresponding oscillator strengths, and the cyclotron absorption spectrum for trilayer graphene are calculated for both $A B A$ and $A B C$ stacking. A gate potential across the stacked layers leads to (1) a reduction of the transition energies, (2) a lifting of the degeneracy of the zero Landau level, and (3) the removal of the electron-hole symmetry.
\end{abstract}

DOI: 10.1103/PhysRevB.86.085412

PACS number(s): 73.22.Pr, 76.40.+b, 81.05.ue

\section{INTRODUCTION}

Monolayer graphene (MG) is a truly two-dimensional (2D) crystal with a gapless linear electronic spectrum at low energy which, along with the chiral nature of its charge carriers is responsible for a variety of unusual properties. ${ }^{1}$ When subjected to a magnetic field $B$, this electronic spectrum evolves into unequally spaced quantized Landau levels (LLs) that display a $\sqrt{B}$ dependence, as well as a zero energy level, in stark contrast with other two-dimensional electron gases. These features were confirmed by means of infrared transmission and cyclotron resonance (CR) experiments. ${ }^{2-5}$ This peculiar behavior of the electron gas in graphene in the presence of a magnetic field is responsible for the anomalous integer quantum Hall effect (IQHE) ${ }^{6-8}$ one of the most remarkable among the new phenomena observed in graphene. Equally interesting, bilayer graphene (BLG) has also shown very unique properties. Presenting a parabolic gapless spectrum at low energies, BLG can be easily converted into a semiconductor with a tunable gap by applying an external electric field that breaks the two layers inversion symmetry. ${ }^{9-11}$ In the presence of a magnetic field this spectrum splits into LLs presenting a rather intricate dependence on Landau level index and magnetic field B. The LLs in BLG move from a linear in $B$ spectrum, characteristic of an ordinary 2D electron gas, to a $\sqrt{B}$ dependence as the energy increases, ${ }^{12,13}$ creating another type of IQHE. ${ }^{14}$

Thicker graphene layers can be also a very attractive subject of research since the stacking order of the layers plays an important role in the features exhibited by the system. Among the multilayer graphene films, trilayer graphene (TLG) has received a lot of attention recently. The two main types of stacking order appearing in nature are the Bernal or $A B A$ stacking, and the rhombohedral or $A B C$ stacking. While $A B A$ TLG presents an electric-field tunable band overlap, the $A B C$ TLG exhibits a tunable band gap. ${ }^{15-19}$ The difference in stacking order also affects the LL behavior in $T L G,{ }^{20,21}$ producing another two new types of IQHE. ${ }^{22,23}$ In the simplest tight-binding model the LL spectrum of $A B A$ TLG is found to be a superposition of a monolayerlike and bilayerlike LL. ${ }^{21,24-27}$ In contrast, the $A B C$ TLG are predicted to have a nearly $B^{3 / 2}$ field dependence..$^{21,24,27,28}$

In the present work we will concentrate on the cyclotron resonance transitions in different stacked TLG. We will compare the results of unbiased and biased layers, and discuss as well the different features appearing due to the stacking order in $A B C$ and $A B A$ TLG. We will concentrate on the position of the cyclotron resonance peaks, and the oscillator strength of those transitions.

The paper is organized as follows. In Sec. II we present the model, calculate the oscillator strength, and present the results for $A B C$ stacking. In Sec. III we consider $A B A$ stacking. The main conclusions are summarized in Sec. IV.

\section{II. $A B C$ STACKING}

Let us consider three coupled graphene layers with an $A B C$ stacking configuration in the continuum approximation. In the present work we retain only the nearest-neighbor coupling terms. In this case, the Hamiltonian can be written as

$$
\mathcal{H}^{A B C}=\left(\begin{array}{cccccc}
U_{1} & v_{F} \pi^{\dagger} & \gamma_{1} & 0 & 0 & 0 \\
v_{F} \pi & U_{1} & 0 & 0 & 0 & 0 \\
\gamma_{1} & 0 & U_{2} & v_{F} \pi & 0 & 0 \\
0 & 0 & v_{F} \pi^{\dagger} & U_{2} & 0 & \gamma_{1} \\
0 & 0 & 0 & 0 & U_{3} & v_{F} \pi^{\dagger} \\
0 & 0 & 0 & \gamma_{1} & v_{F} \pi & U_{3}
\end{array}\right),
$$

whose eigenstates are defined by the six components spinor $\Psi=\left[\psi_{A_{1}}, \psi_{B_{1}}, \psi_{B_{2}}, \psi_{A_{2}}, \psi_{A_{3}}, \psi_{B_{3}}\right]^{T}$. In the above Hamiltonian $\pi \equiv p_{x}+i p_{y}, \pi^{\dagger} \equiv p_{x}-i p_{y}, v_{F}=\sqrt{3} a \gamma_{0} /(2 \hbar)$ is the Fermi velocity in terms of the in-plane nearest neighbor hopping $\gamma_{0}=3.12 \mathrm{eV}$, and the carbon-carbon distance $a=$ $1.42 \AA . U_{i}(i=1,2,3)$ is the potential in each layer which we consider constant throughout each layer, and $\gamma_{1}=0.4 \mathrm{eV}$ is the nearest neighbor coupling term between adjacent layers. In the presence of a magnetic field $\mathbf{p} \rightarrow \mathbf{p}+|e| \mathbf{A}$, where $e$ is the electron charge. By making use of the Landau gauge $\mathbf{A}=B(0, x, 0)$, we have $\left[p_{y}, H\right]=0$ and the eigenstates are found to be

$$
\begin{aligned}
& \Psi_{n}^{A B C}=A_{n}\left[i d_{n} \psi_{n+1}, \frac{-d_{n}}{\delta_{1}^{n} l_{B}} \psi_{n+2}, i c_{n} \psi_{n+1},\right. \\
& \left.b_{n} \psi_{n}, \frac{-i 2 n}{\delta_{3}^{n} l_{B}} \psi_{n-1}, \psi_{n}\right]^{T},
\end{aligned}
$$

with $\psi_{n}$ given by

$$
\psi_{n}=e^{i k_{y} y} e^{-\bar{x}^{2} / 2} H_{n}(\bar{x}),
$$

where $\bar{x} \equiv x / l_{B}+l_{B} k_{y}, l_{B} \equiv \sqrt{\hbar / e B}$ is the magnetic length, $\delta_{i}^{n} \equiv\left(E_{n}-U_{i}\right) /\left(\hbar v_{F}\right), H_{n}(\bar{x})$ is the Hermite polynomial, and 

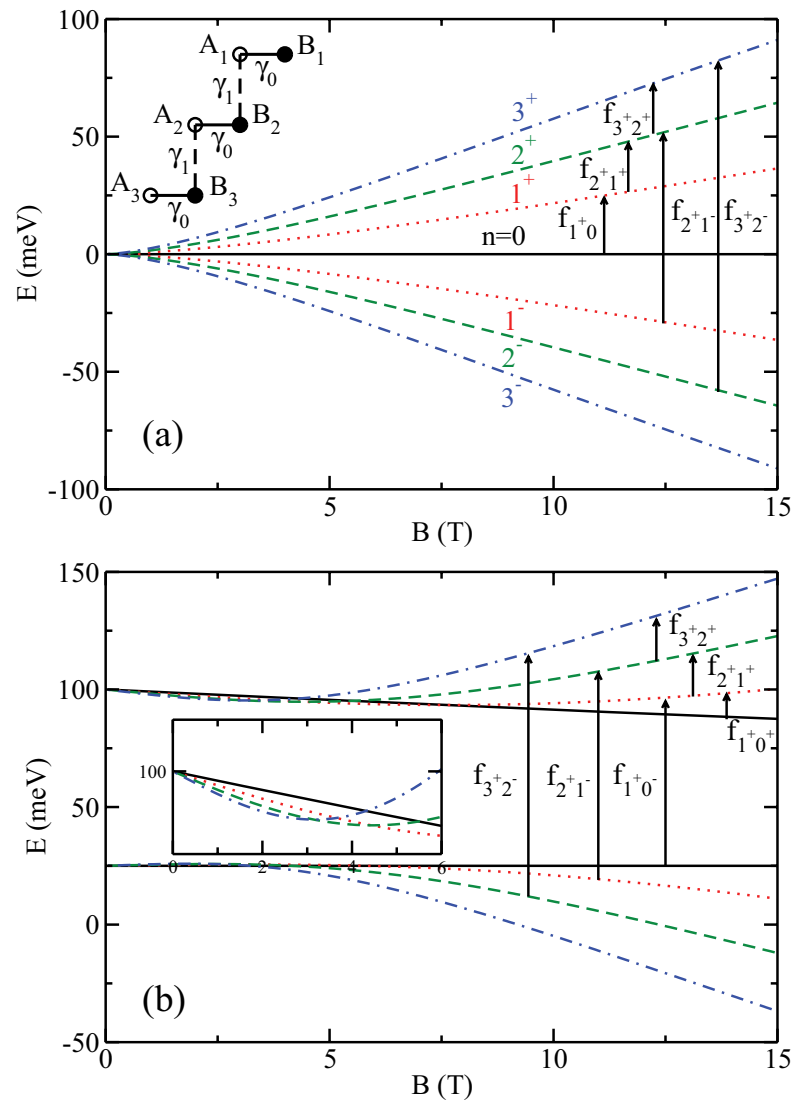

FIG. 1. (Color online) TLG Landau-level spectrum as a function of magnetic field for $A B C$ stacking with (a) $U_{1}=U_{2}=U_{3}=0$, and (b) $U_{1}=100 \mathrm{meV}, U_{2}=50 \mathrm{meV}, U_{3}=25 \mathrm{meV}$ for $n=0$ (black solid lines), $n=1$ (red dotted lines), $n=2$ (green dashed lines), and $n=3$ (blue dot-dashed lines). The inset in (a) shows the coupling between the three layers, while in (b) it shows an enlargement of the LLs crossings for low magnetic fields. The vertical arrows indicate some of the allowed optical transitions.

$\psi_{n} \equiv 0$ for $n<0$. The other constants appearing in $\Psi_{n}^{A B C}$ are defined in Appendix A.

The magnetic field dependence of the LL are shown in Fig. 1. The levels for unbiased layers are shown in Fig. 1(a) where they behave as $B^{3 / 2}$ for low fields and become linear in $B$ for large fields. ${ }^{21}$ When a bias is present, we show as an example in Fig. 1(b) the LL for $U_{1}=100 \mathrm{meV}, U_{2}=50 \mathrm{meV}$, and $U_{3}=25 \mathrm{meV}$. The arrows indicate some of the allowed transitions dictated by the dipole selection rule.

The oscillator strength (OS) of the transition between the initial state $\left|\Psi_{n}\right\rangle$ and the final one $\left|\Psi_{m}\right\rangle$ is usually defined within the dipole approximation in terms of $\left|\left\langle\Psi_{m}|x| \Psi_{n}\right\rangle\right|^{2}$. Since the only effect of $k_{y}$ is to shift the symmetry center of the wave function and the system is assumed to be infinitely extended, we set $k_{y}=0$ and work with the dimensionless quantity

$f_{m n}=\frac{1}{l_{B}^{2}}\left|\int \Psi_{m}^{*} x \Psi_{n} d x\right|^{2}=l_{B}^{2}\left|\sum_{C} \int \Psi_{C}^{m *} \bar{x} \Psi_{C}^{n} d \bar{x}\right|^{2}$,

with $C=A_{1}, B_{1}, B_{2}, A_{2}, A_{3}, B_{3}$ that are defined in the inset of Fig. 1(a). The analytical results of these integrals are given in
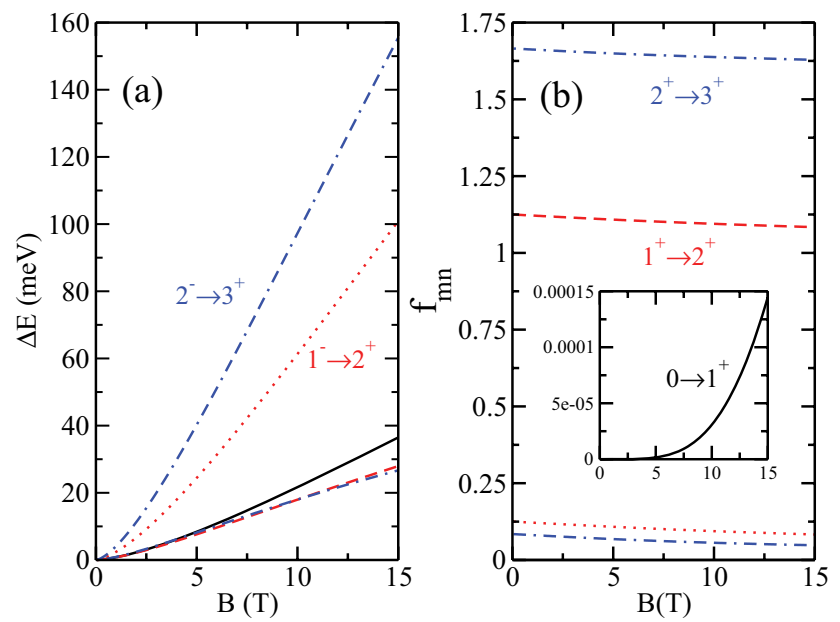

FIG. 2. (Color online) Left panel (a) shows the transition energies as a function of the magnetic field $B$ for unbiased, i.e., $U_{1}=U_{2}=$ $U_{3}=0, A B C$ layers. The right panel (b) presents the oscillator strength of the allowed transitions for the low-lying energy Landau levels.

Appendix A. Throughout the paper we will refer to a transition from the state $\left|\Psi_{n}\right\rangle$ to $\left|\Psi_{m}\right\rangle$ as $(n, m)$.

Figure 2(a) shows the most important energy transitions $\triangle E$ in $A B C$ TLG as a function of the magnetic field for the levels displayed in Fig. 1(a). The black solid, red dashed, and blue dot-dashed lines present the intraband transitions $\left(0,1^{+}\right),\left(1^{+}, 2^{+}\right)$, and $\left(2^{+}, 3^{+}\right)$, while the red dotted and blue dash-dash-dotted lines show the interband transitions $\left(1^{-}, 2^{+}\right)$and $\left(2^{-}, 3^{+}\right)$, respectively. Here the sign "+"("-") stands for electron (hole) states. The transitions follow a $B^{3 / 2}$ dependence for low magnetic fields $(B<3 \mathrm{~T})$ and this behavior turns into a linear $B$ dependence for higher values of the field $(B>10 \mathrm{~T})$. Figure 2(b) shows the corresponding oscillator strength as a function of the magnetic field for the same transitions appearing in the left panel. All transitions show an oscillator strength that monotonically decreases with increasing magnetic field with the exception of the transition involving the zero LL [see inset of Fig. 2(b)]. Notice that the OS associated with interband transitions are much smaller than those involving the intraband transitions.

The effect of an applied bias is explored in Fig. 3, where we show the energy transitions (a) and oscillator strength (b) as function of magnetic field for biased layers with $U_{1}=$ $100 \mathrm{meV}, U_{2}=50 \mathrm{meV}$, and $U_{3}=25 \mathrm{meV}$. The small difference between the transition energies of the dashed and dotted red (green) lines reveals a clear asymmetry between electron and hole transitions. For low magnetic fields, the energies involved in the intraband electron transitions are slightly negative due to the crossing between the LLs shown in the inset of Fig. 1(b), which are also responsible for the crossings happening between the interband energy transitions (solid lines) in the left panel of Fig. 3. In contrast to the case with unbiased layers, now the OS show a stronger dependence on the magnetic field, and although the electron and hole have very similar intraband energy transitions, their OS are remarkably different and have the opposite magnetic field dependence. While the holelike transitions $\left(2^{-}, 1^{-}\right),\left(3^{-}, 2^{-}\right)$gain in OS as the magnetic field increases, the electronlike transitions 

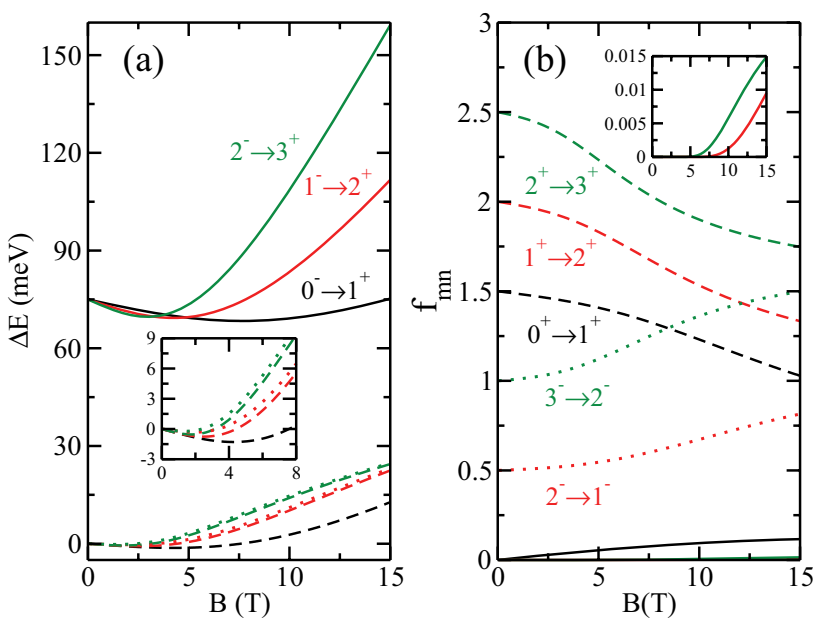

FIG. 3. (Color online) (a) Transition energies between the lowlying energy $\mathrm{LL}$ in a biased $A B C$ TLG as a function of the magnetic field for $U_{1}=100 \mathrm{meV}, U_{2}=50 \mathrm{meV}$, and $U_{3}=25 \mathrm{meV}$. (b) Oscillator strength vs magnetic field $B$ for the transitions presented in (a).

$\left(1^{+}, 2^{+}\right),\left(2^{+}, 3^{+}\right)$experience a considerable decrease in OS. The inset in Fig. 3(b) shows the very small OS of the transitions $\left(1^{-}, 2^{+}\right)$and $\left(2^{-}, 3^{+}\right)$.

Figure 4 shows the TLG Landau-level spectrum as function of $U_{1}$ (with $U_{2}=U_{3}=0$ ) (a) and $U_{3}$ (with $U_{1}=U_{2}=0$ ) (b) for $n=0$ (black solid lines), $n=1$ (red dotted lines), $n=2$ (green dashed lines), and $n=3$ (blue dot-dashed lines) for a fixed magnetic field of $10 \mathrm{~T}$. Among the differences between the panels (a) and (b) it can be noticed that the energies increase faster with $U_{3}$ and also for $n=0$ we have that $E=U_{3}$ is always an eigenenergy, although $E=U_{1}$ is not. Figure 5 shows the transition energies between some of the levels appearing in Fig. 4. It also shows the behavior of the oscillator strength as $U_{1}$ (top left panel) and $U_{3}$ (top right panel) increase. The inset in top-right panel shows the transition
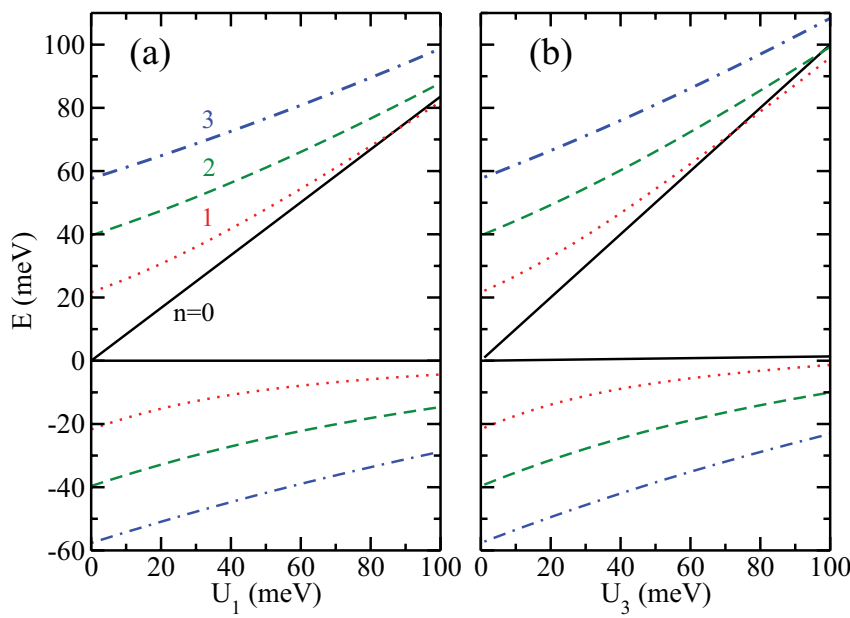

FIG. 4. (Color online) TLG Landau-level spectrum with a fixed magnetic field of $10 \mathrm{~T}$ for the $A B C$ stacking as a function of $U_{1}$ with $U_{2}=U_{3}=0$ (a) and $U_{3}$ with $U_{1}=U_{2}=0$ (b) for $n=0$ (black solid lines), $n=1$ (red dotted lines), $n=2$ (green dashed lines), and $n=3$ (blue dot-dashed lines).
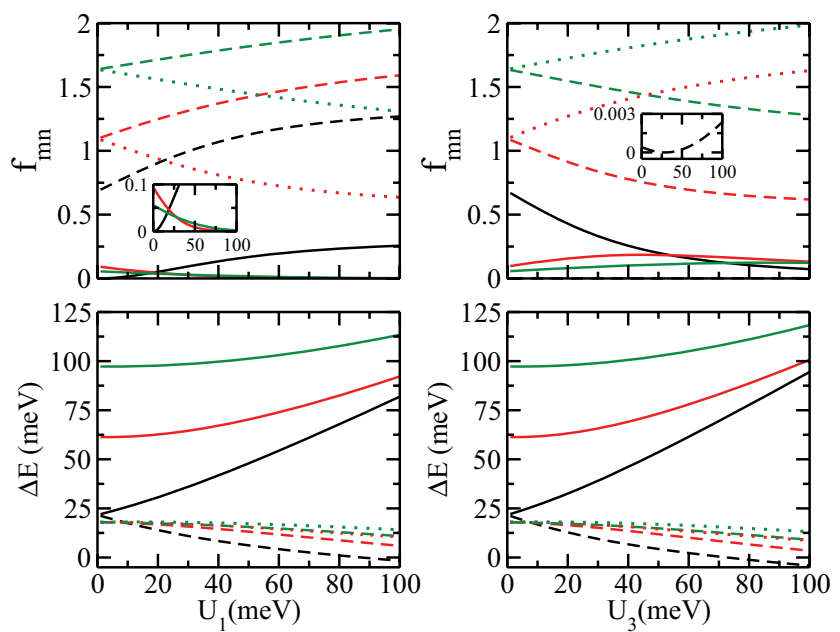

FIG. 5. (Color online) Energy transitions (bottom panels) and oscillator strength (top panels) as a function of $U_{1}$ and $U_{3}$ for fixed magnetic field of $10 \mathrm{~T}$. The potential in the other two layers is kept zero. The inset in the top-left panel shows the transitions $\left(1^{-}, 2^{+}\right)$ (red solid line) and $\left(2^{-}, 3^{+}\right)$(green solid line), while the inset in the top-right panel shows the transition $\left(0^{+}, 1^{+}\right)$(black dashed line).

$\left(0^{+}, 1^{+}\right)$which has a very small OS as compared to the other ones. The legend for this graph is the same as in Fig. 3. The transition energies shown in the bottom panels are very similar, but when $U_{3}$ is increased the interband (intraband) transitions occur at slightly higher (lower) energies than when $U_{1}$ is increased. The difference in the role played by the top and bottom layers can be better noticed when we look at the oscillator strengths, which behave completely different whether we vary $U_{1}$ or $U_{3}$. Among the discrepancies, we can highlight the transition $\left(0^{-}, 1^{+}\right)$(black solid line) that presents a small monotonic increase with $U_{1}$ and an accentuated decrease with $U_{3}$. It is also worth noticing the holelike transitions $\left(2^{-}, 1^{-}\right)$(red dotted line) and $\left(3^{-}, 2^{-}\right)$(green dotted line), that monotonically decrease as $U_{1}$ increases, and show the opposite behavior as we increase $U_{3}$. On the other hand, the electronlike transitions $\left(1^{+}, 2^{+}\right)$(red dashed line) and $\left(2^{+}, 3^{+}\right)$ (green dashed line) decrease as $U_{3}$ is increased, while the increase of $U_{1}$ causes a gain in their OS.

For completeness we also calculated the CR spectrum as

$$
\sigma(E)=\sum_{n, m} \frac{(\Gamma / 2 \pi) E_{m n} f_{m n}}{\left(E-E_{m n}\right)^{2}+\Gamma^{2} / 4} f\left(E_{n}\right)\left[1-f\left(E_{m}\right)\right],
$$

where $E_{m n}=E_{m}-E_{n}$ is the transition energy, $f_{m n}$ is the OS defined in Eq. (3), $\Gamma$ is the broadening of the Lorentzian function, and $f\left(E_{n}\right)$ is the Fermi-Dirac distribution. In the numerical results we set the temperature equal to zero and $\Gamma=$ $2 \mathrm{meV}$. The position of the Fermi level $E_{F}$ is taken between the levels $0\left(0^{+}\right)$and $1^{+}\left(1^{+}\right)$for the unbiased (biased) case.

Figure 6 shows the optical absorption peaks for $A B C$ TLG. Panels (a) and (b) correspond to the unbiased case for $B=10 \mathrm{~T}$ (black line) and $B=15$ (red line) T, while the panels (c) and (d) present the absorption for biased layers, respectively. Notice in panel (a) the absence of the peak corresponding to the transition $(0,1)$ (around $E=22 \mathrm{meV}$ ), which is caused by the small value of the OS of this transition [see Fig. 2(b)] that makes the contributions of the other transitions outgrow the 

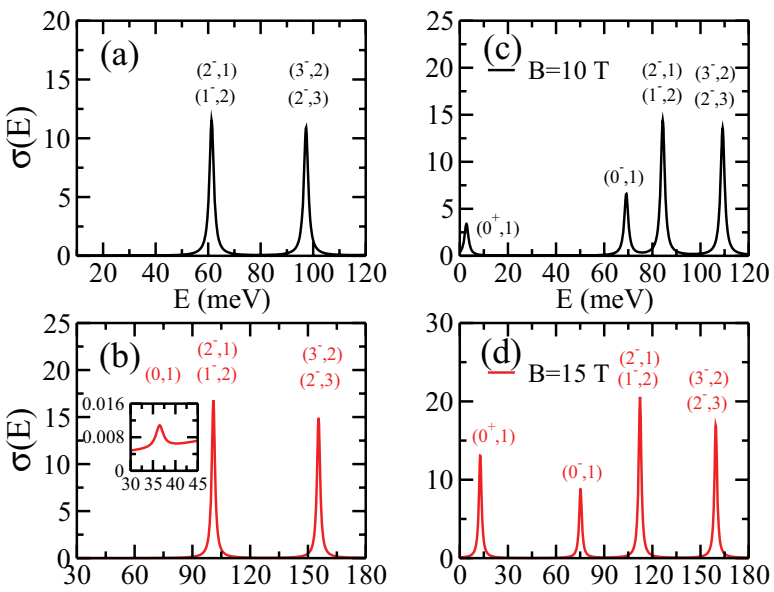

FIG. 6. (Color online) $\sigma(E)$ vs energy in $A B C$ TLG for $B=$ $10 \mathrm{~T}$ (black lines) and $B=15 \mathrm{~T}$ (red lines). The left (right) panels correspond to unbiased layers (biased). The inset shows the first absorption peak corresponding to the transition $(0,1)$.

absorption peak in this region of energy. In panel (b) this first transition peak is now present around $36.5 \mathrm{meV}$, although very small compared to the other absorption peaks. When a bias is applied we observe the presence of an extra peak due the lifting of the electron-hole degeneracy of the $n=0 \mathrm{LL}$.

\section{ABA STACKING}

Let us now turn our attention to the case of $A B A$ stacking. The Hamiltonian for $A B A$-stacked TLG around the K point is given by

$$
\mathcal{H}^{A B A}=\left(\begin{array}{cccccc}
U_{1} & v_{F} \pi^{\dagger} & \gamma_{1} & 0 & 0 & 0 \\
v_{F} \pi & U_{1} & 0 & 0 & 0 & 0 \\
\gamma_{1} & 0 & U_{2} & v_{F} \pi & \gamma_{1} & 0 \\
0 & 0 & v_{F} \pi^{\dagger} & U_{2} & 0 & 0 \\
0 & 0 & \gamma_{1} & 0 & U_{3} & v_{F} \pi^{\dagger} \\
0 & 0 & 0 & 0 & v_{F} \pi & U_{3}
\end{array}\right) .
$$

In the presence of a magnetic field the six-component spinor of the $n$th LL is given by

$$
\begin{array}{r}
\Psi_{n}^{A B A}=B_{n}\left[-i l_{B} \delta_{1}^{n} \psi_{n-1}, \psi_{n}, i b_{1}^{n} \psi_{n-1}, \frac{2(n-1) b_{1}^{n}}{l_{B} \delta_{2}^{n}} \psi_{n-2},\right. \\
\left.\frac{-i l_{B} \delta_{3}^{n} b_{1}^{n}}{b_{3}^{n}} \psi_{n-1}, \frac{b_{1}^{n}}{b_{3}^{n}} \psi_{n}\right]^{T}
\end{array}
$$

for $n>0$, where $b_{1(3)}^{n}$ are given in Appendix B. It is worth highlighting that in the case of unbiased layers the LLs are simply the superposition of the monolayer and bilayer spectrum, and the spinor components that correspond to the third layer are identical to the ones of the first layer, as it should be since the top and bottom layers are then symmetric relative to the middle layer. For this reason, we will refer now to the Landau index no longer as $n$, but as $n_{m}=n$ for the monolayerlike levels and as $n_{b}=n-1$ for the bilayerlike levels. The case $n_{m}=0$ generates two LL, as can be seen in

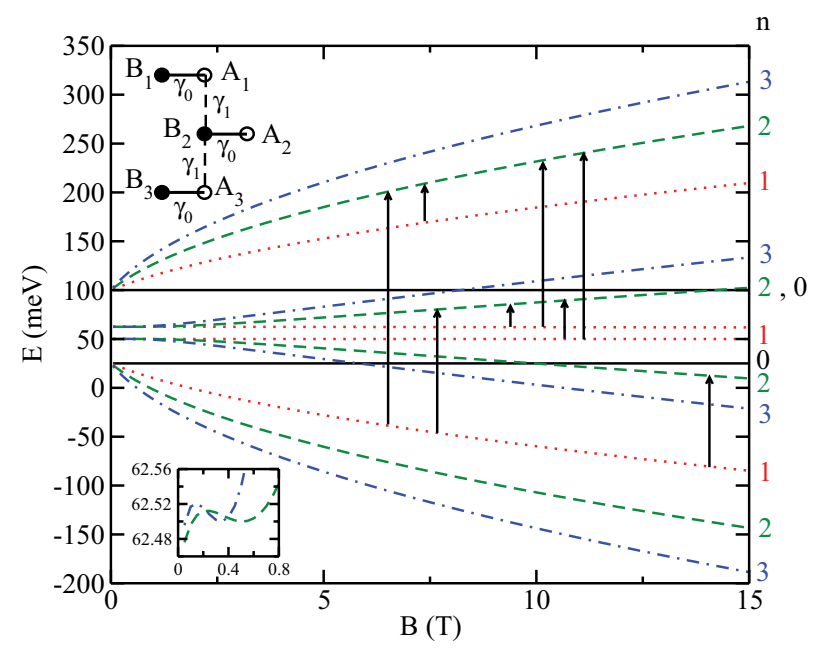

FIG. 7. (Color online) $A B A$ TLG Landau-level spectrum as a function of $B$ with $U_{1}=100 \mathrm{meV}, U_{2}=50 \mathrm{meV}$, and $U_{3}=25 \mathrm{meV}$ for $n=0$ (black solid lines), $n=1$ (red dotted lines), $n=2$ (green dashed lines), and $n=3$ (blue dot-dashed lines). The upper inset shows the coupling between the layers and the lower inset shows an enlargement of the nonlinear behavior of the levels $n_{b}=1^{+}, 2^{+}$for low $B$.

Fig. 7, the level we call $0^{+}$corresponds to $E_{0}=U_{1}$, and for the level $0^{-}, E_{0}=U_{3}$. The spinor associated to these zero levels are $\Psi_{0^{+}}^{A B A}=1 /\left(l_{B} \sqrt{\pi}\right)^{1 / 2}\left[\begin{array}{llllll}0, & \psi_{0}, & 0, & 0, & 0, & 0\end{array}\right]$ and $\Psi_{0^{-}}^{A B A}=1 /\left(l_{B} \sqrt{\pi}\right)^{1 / 2}\left[0,0,0,0,0, \psi_{0}\right]$, respectively, while $n_{b}=0(n=1)$ produces $E_{1}=U_{2}$ and a level starting from $E_{1}=\left(U_{1}+U_{3}\right) / 2$ that presents a small monotonic decrease with $B$.

The oscillator strength was calculated according to Eq. (3) and the explicit expressions for the $A B A$ stacking are given in Appendix B. Figure 8(a) shows the energies of some possible transitions between monolayerlike levels of the unbiased layers. All these transitions occur at energies that follow a $\sqrt{B}$ dependence. The oscillator strengths related to these
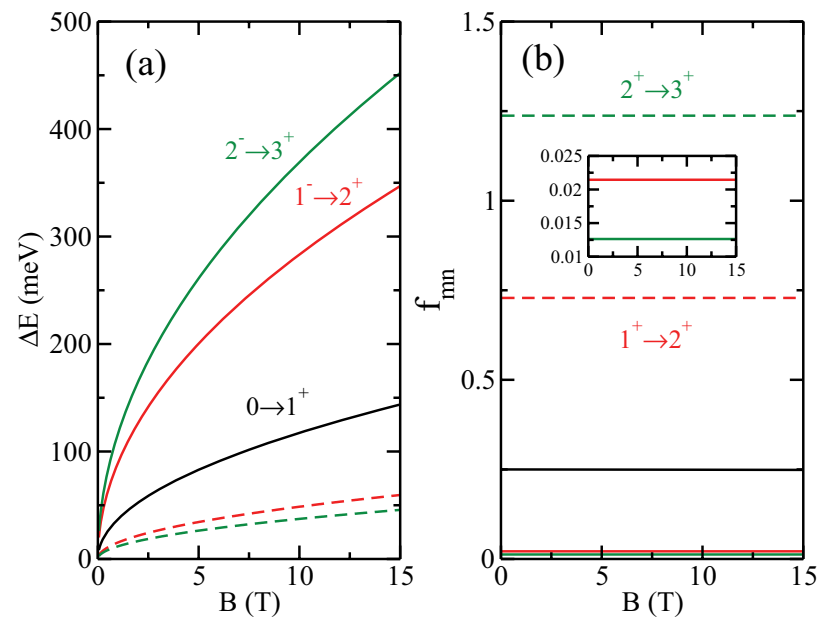

FIG. 8. (Color online) (a) Transition energies between monolayerlike levels as a function of the magnetic field $B$ for unbiased, $U_{1}=U_{2}=U_{3}=0, A B A$ layers. (b) The corresponding oscillator strengths are shown in the left panel. 

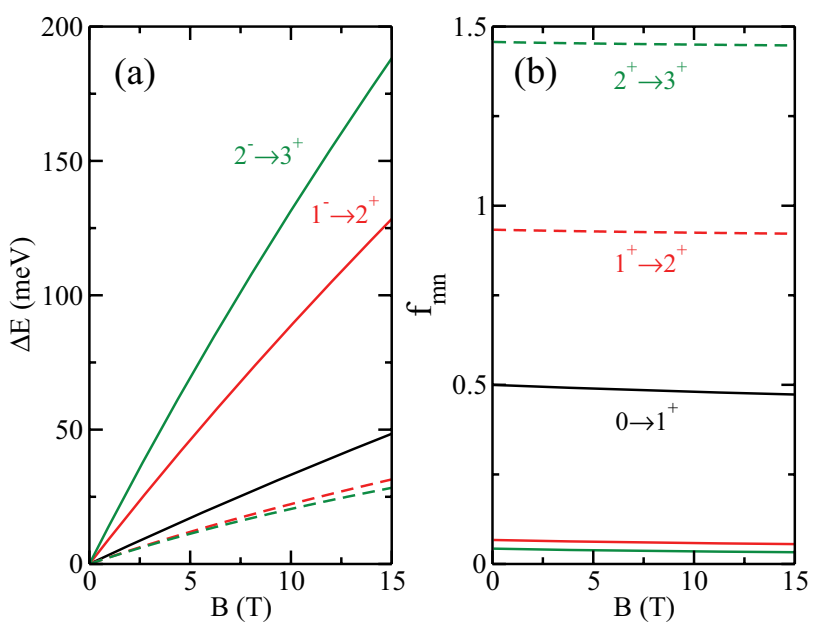

FIG. 9. (Color online) (a) Transition energies between bilayerlike levels as a function of the magnetic field $B$ for unbiased, $U_{1}=U_{2}=$ $U_{3}=0, A B A$ layers. (b) The oscillator strength for the transitions shown in the left panel.

transitions are presented in Fig. 8(b), where we can see that the transition involving the zero energy level is the most pronounced one, and the interband transitions are very small [see inset of Fig. 8(b)]. Figure 9 presents the same kind of results, but now for transitions between levels characteristic of the bilayer system. These transition energies are linear in $B$ for low values of the magnetic field $(B<5 \mathrm{~T})$, however, as $B$ increases the linear behavior turns into a $\sqrt{B}$ dependence. The OS of the bilayerlike transitions present all the same kind of behavior, i.e., a weak monotonic decrease as $B$ increases.

In Fig. 10 the transitions between monolayerlike and bilayerlike levels are presented. The transition energy $\left(n_{b}=\right.$ $0, n_{m}=2^{+}$), black solid curve, follows a perfect $\sqrt{B}$ dependence, since the level $n_{b}=0$ is simply $E=0$. The energies correspond to the transition $\left(n_{m}=1^{-}, n_{b}=1^{+}\right)$, red solid line, $\left(n_{b}=1^{-}, n_{m}=3^{+}\right)$, green solid line, and $\left(n_{m}=2^{-}, n_{b}=2^{+}\right)$, green dashed line, can be well fitted by a power law with
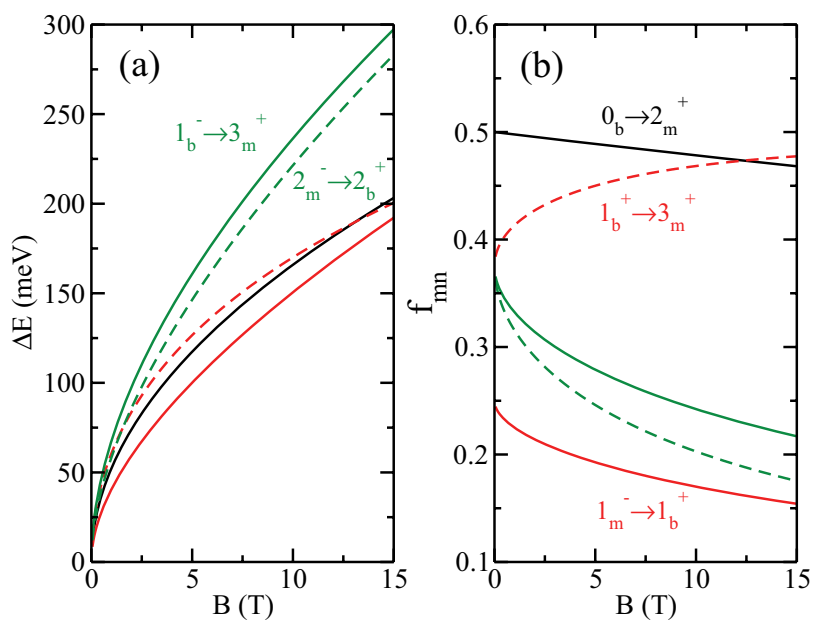

FIG. 10. (Color online) (a) Transition energies between monolayerlike and bilayerlike levels as a function of the magnetic field $B$ for unbiased, $U_{1}=U_{2}=U_{3}=0, A B A$ layers. (b) The oscillator strength for the transitions shown in the left panel.
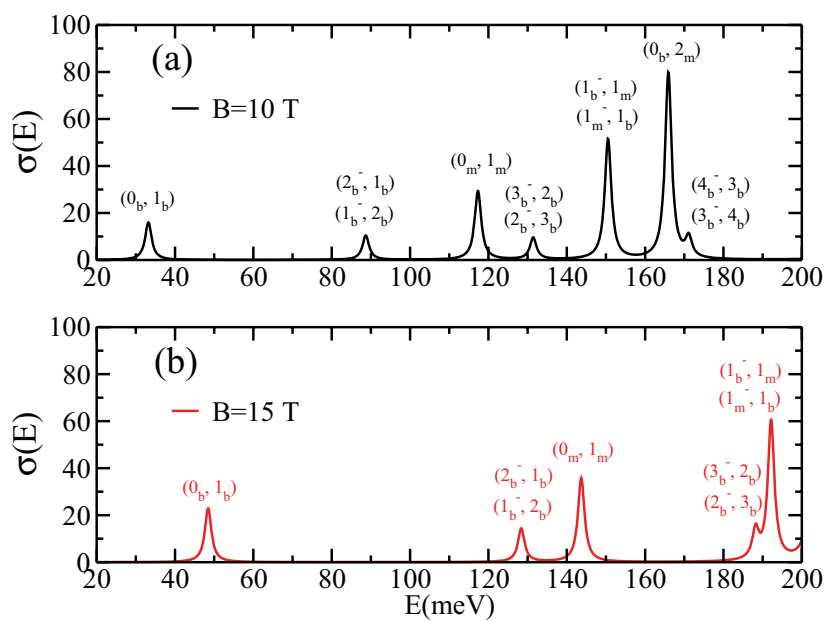

FIG. 11. (Color online) $\sigma(E)$ vs energy in an unbiased $A B A$ TLG for $B=10 \mathrm{~T}$ (black lines) and $B=15 \mathrm{~T}$ (red lines).

an exponent that varies from 0.5 (low fields) to 0.6 (high fields) as $B$ increases. The transition $\left(n_{b}=1^{+}, n_{m}=3^{+}\right)$can be also fairly approximated by a power law, but in this case the exponent varies from 0.4 to 0.5 as the magnetic field increases. The OS are shown in panel (b) where we can see that all OS decrease with increasing $B$ with the exception of the intraband transition $\left(n_{b}=1^{+}, n_{m}=3^{+}\right)$, red dashed line, which exhibits the opposite behavior.

The CR absorption peaks of an unbiased $A B A$ TLG are displayed in Fig. 11 for $B=10 \mathrm{~T}$ [panel (a)] and $B=15 \mathrm{~T}$ [panel (b)]. The Fermi energy is taken to be $E=0$. Above each peak it is shown which transitions are involved. We can notice that with the exception of the peaks associated to transitions involving the level $E=0$ each peak receives equal contributions of two interband transitions. It means that if, for instance, the Fermi level is increased in order to cross the level $n_{b}=1^{+}$, the first and third peaks will disappear, the second and fifth peaks will drop to half of their values, and the other ones will be unaffected.

The following results consider the effect of a bias $\left(U_{1}=\right.$ $100 \mathrm{meV}, U_{2}=50 \mathrm{meV}$, and $U_{3}=25 \mathrm{meV}$ ) that breaks the mirror reflection symmetry of the $A B A$ TLG. Figure 12(a) shows some of the transitions between the monolayerlike levels. We see two groups of transition energies: the intraband transitons (dashed and dotted lines starting from $E=0$ ) and interband transitions (solid lines starting from $E=75 \mathrm{meV}$ ). The inset displays the small asymmetry between the electronlike $\left(n_{m}=0^{+}, n_{m}=1^{+}\right)$and holelike $\left(n_{m}=1^{-}, n_{m}=0^{-}\right)$ transitions due to the bias. In general, the transition energies increase less fast with $B$ when a bias is applied. In panel (b) we notice that the OS of all the intraband transitions have the same decreasing behavior as $B$ increases, exhibiting a more strong dependence on $B$ up to $5 \mathrm{~T}$. Remarkably, the OS of the interband transitions exhibit the opposite behavior, i.e., a small monotonically increasing behavior with $B$. The upper inset displays an enlarged view of the OS for the transitions $\left(n_{m}=1^{-}, n_{m}=2^{+}\right)$and $\left(n_{m}=2^{-}, n_{m}=3^{+}\right)$.

Figure 13 focus on the transitions between bilayerlike levels. The main effect of the bias on the transition energies is the disappearance of the linear behavior for small values of $B$ (see the inset of Fig. 7) and the breaking of the symmetry 

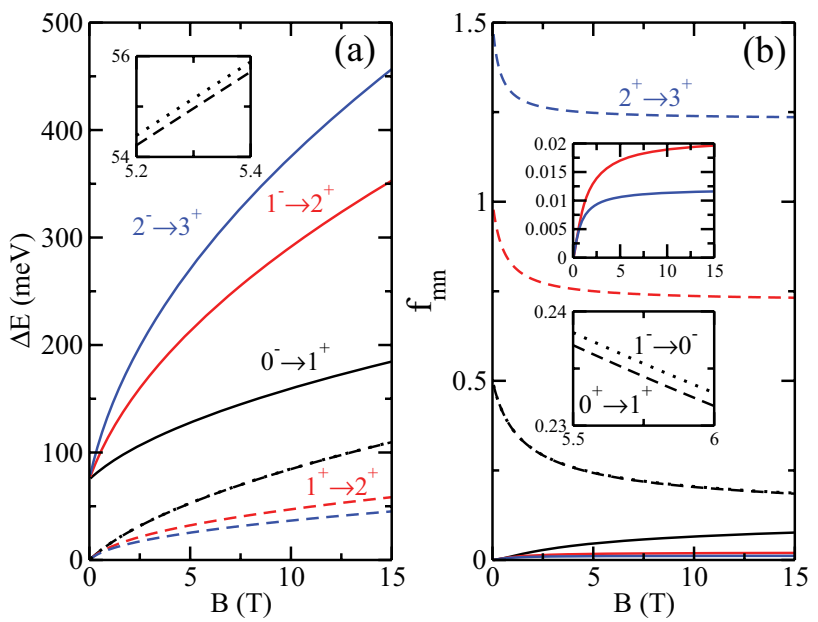

FIG. 12. (Color online) (a) Transition energies between monolayerlike levels as a function of the magnetic field $B$ for biased $A B A$ layers. The inset shows the small difference in energy of the electron and holelike transitions. (b) The oscillator strength for the transitions shown in the left panel. The lower inset shows the small difference in OS of the transitions $\left(n_{m}=0^{+}, n_{m}=1^{+}\right)$and $\left(n_{m}=1^{-}, n_{m}=0^{-}\right)$, while the upper inset shows an enlargement of the $\left(n_{m}=1^{-}, n_{m}=2^{+}\right)$and $\left(n_{m}=2^{-}, n_{m}=3^{+}\right)$.

between electrons and holes, as shown in the inset of panel (a). In panel (b) we see that for all the transitions, with the exception of $\left(n_{b}=1^{-}, n_{b}=2^{+}\right)$, the OS exhibit a strong dependence on $B$, especially for low fields. The OS for the holelike transitions have a strong increase up to $5 \mathrm{~T}$ and then they continue to increase but now less fast, while for the electronlike transitions the OS presents a significant increase for small values of $B$ with a subsequent decrease.

Figure 14 exhibits some transitions from monolayer to bilayerlike levels. The energy of the transition $\left(n_{m}=0^{-}, n_{b}=\right.$ $0^{-}$) is $25 \mathrm{meV}$ and constant while its OS exhibits a strong magnetic field dependence [panel (b)] due to the magnetic length
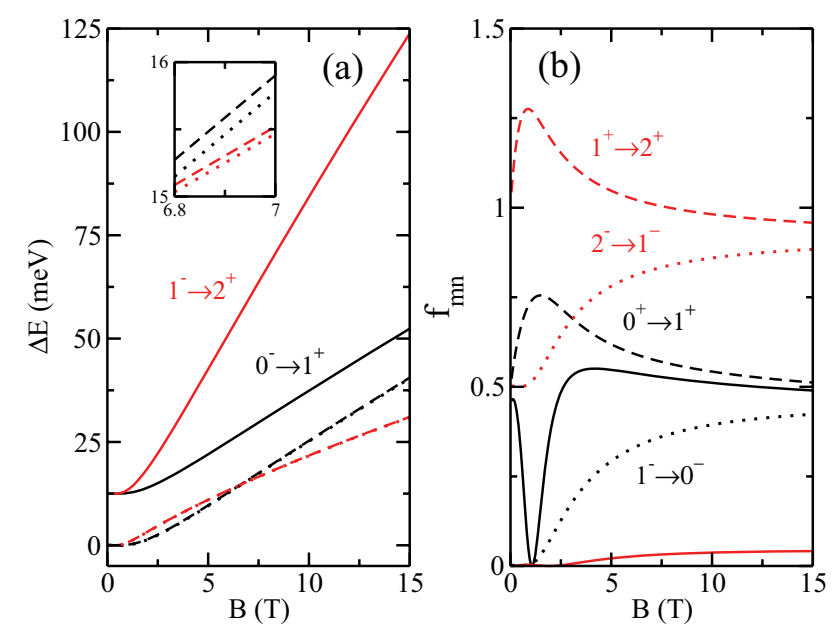

FIG. 13. (Color online) (a) Transition energies between bilayerlike levels as a function of the magnetic field $B$ for biased $A B A$ layers. The inset shows the small difference in energy of the electron and holelike transitions. (b) The oscillator strength for the transitions are shown in the left panel.
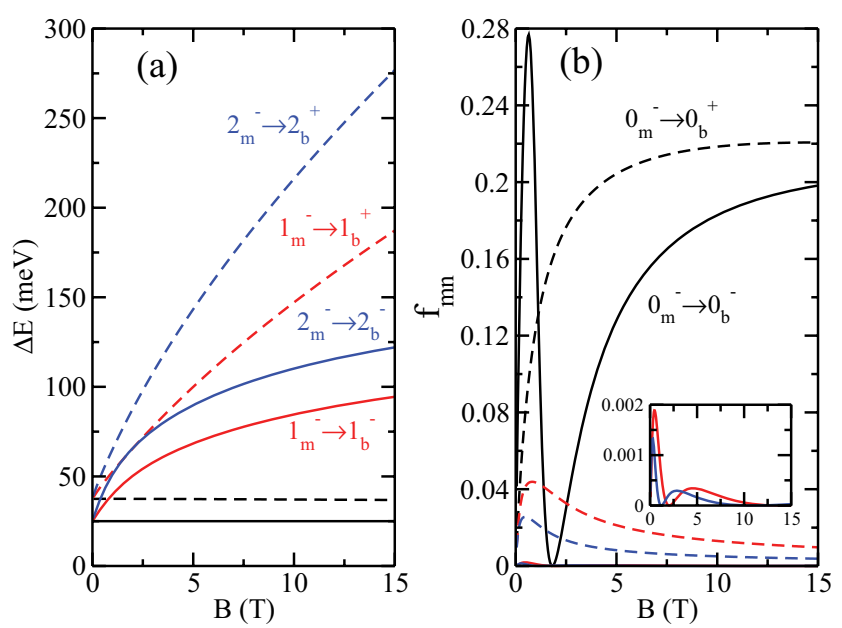

FIG. 14. (Color online) (a) Transition energies from monolayer to bilayerlike levels as a function of the magnetic field $B$ for biased $A B A$ layers. (b) The oscillator strength for the transitions shown in the left panel. The inset shows the OS of the transitions $\left(n_{m}=1^{-}, n_{b}=1^{-}\right)$ in red solid line and $\left(n_{m}=2^{-}, n_{b}=2^{-}\right)$in blue solid line.

$l_{B}$ appearing in the constants of Appendix B. The other two intraband transition energies (red and blue solid curves) can be well fitted by a power law whose exponent approaches 0.3 and the oscillator strengths of these transitions are very small as shown in the inset of panel (b). The interband transitions $\left(n_{m}=1^{-}, n_{b}=1^{+}\right)$, red dashed line, and $\left(n_{m}=2^{-}, n_{b}=2^{+}\right)$, blue dashed line, have energies that can be described by a $B^{0.6}$ dependence for $B>5 \mathrm{~T}$ and their OS have a small increase for low values of magnetic field followed by a monotonic decrease as can be seen in panel (b). In Fig. 15 we present the same kind of results for some transitions from bilayer to monolayerlike levels. Note that in this case, with the exception of the intraband transition $\left(n_{b}=0^{+}, n_{m}=0^{+}\right)$, all the transitions have low oscillator strengths for fields higher than $3 \mathrm{~T}$.
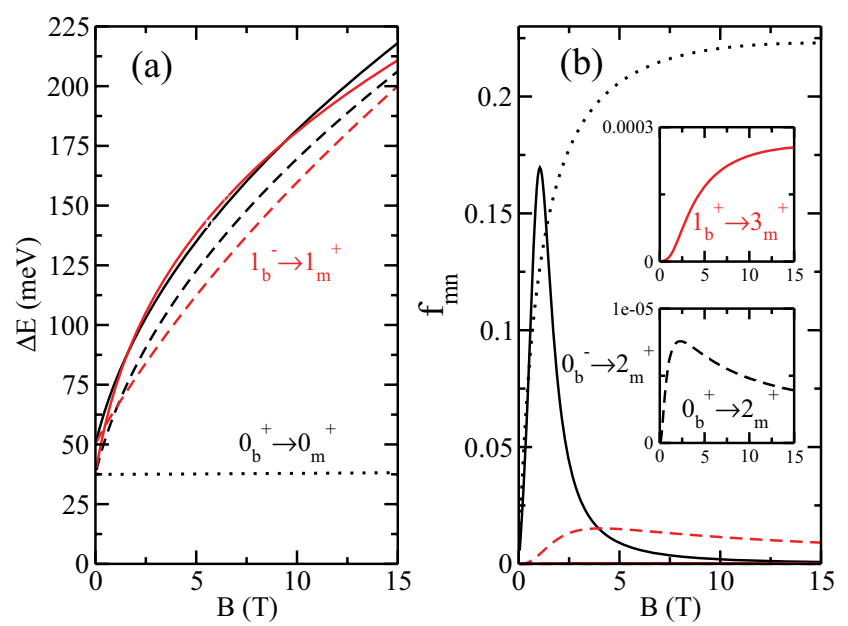

FIG. 15. (Color online) (a) Energies of some of the transitions from bilayer to monolayerlike levels as a function of the magnetic field $B$ for biased $A B A$ layers. (b) The oscillator strength for the transitions shown in the left panel. The lower inset displays the OS of the transition $\left(n_{b}=0^{+}, n_{m}=2^{+}\right)$in black dashed line, while the upper inset displays the transitions $\left(n_{b}=1^{-}, n_{m}=3^{+}\right)$and $\left(n_{b}=\right.$ $\left.1^{+}, n_{m}=3^{+}\right)$. 

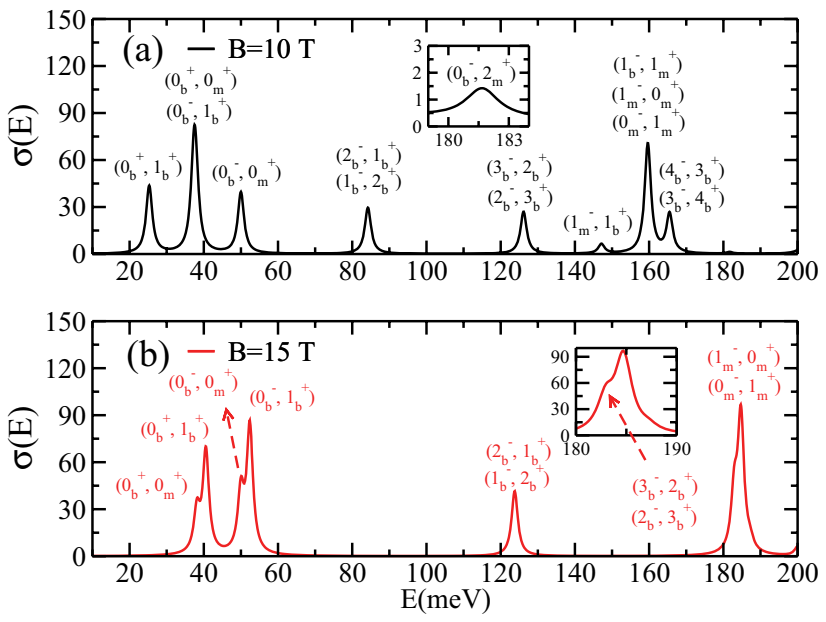

FIG. 16. (Color online) $\sigma(E)$ vs energy in a biased $A B A\left(U_{1}=\right.$ $100 \mathrm{meV}, U_{2}=50 \mathrm{meV}, U_{3}=25 \mathrm{meV}$ ) TLG for $B=10 \mathrm{~T}$ (black lines) and $B=15 \mathrm{~T}$ (red lines). The inset in (a) shows an enlargement of the transition occurring around $181.5 \mathrm{meV}$, while the inset panel (b) shows the formation of a double peak around $183 \mathrm{meV}$ and $184.8 \mathrm{meV}$.

Figure 16 shows the $C R$ spectrum for the biased $A B A$ layers for $B=10 \mathrm{~T}$ [panel (a) ] and $B=15 \mathrm{~T}$ [panel (b)]. We assumed that all levels are occupied until $0_{b}^{+}$. This result summarizes the main effects of the bias on the transitions between the LL, since it takes into account both transition energies and oscillator strength. We observe more CR lines at low energies due to the lifting of the fourfold degeneracy (without taking into account spin and valley degeneracy) of the level 0 . The asymmetry between electrons and holes induced by the bias are usually small, which makes, for instance, the transitions $\left(n_{b}=2^{-}, n_{b}=1^{+}\right)$and $\left(n_{b}=1^{-}, n_{b}=2^{+}\right)$appear as a single $\mathrm{CR}$ line, since the difference in energy of these transitions are smaller than the broadening $\Gamma$ of the peaks. The only evidence of this kind of asymmetry in the CR spectrum is given by the transitions $\left(n_{m}=1^{-}, n_{b}=1^{+}\right)$and $\left(n_{b}=1^{-}, n_{m}=1^{+}\right)$ which occur at $147.14 \mathrm{meV}$ and $159.69 \mathrm{meV}$ for $B=10 \mathrm{~T}$, respectively. The inset in panel (a) shows an enlargement of the CR line corresponding to $\left(n_{b}=0^{-}, n_{m}=2^{+}\right)$around $181.5 \mathrm{meV}$. Note also that the transition $\left(n_{b}=0^{+}, n_{m}=2^{+}\right)$ is not visible since its OS is very small [see inset in Fig. 15(b)]. In panel (b) it can be seen that the first three peaks in panel (a) become two double peaks, since the energies of the transitions $\left(n_{b}=0^{+}, n_{m}=0^{+}\right)$and $\left(n_{b}=0^{-}, n_{m}=0^{+}\right)$do not change, and the energies of $\left(n_{b}=0^{+}, n_{b}=1^{+}\right)$and $\left(n_{b}=0^{-}, n_{b}=\right.$ $\left.1^{+}\right)$increase. A similar behavior occurs around $185 \mathrm{meV}$; the transition energies of $\left(n_{b}=2^{-}, n_{b}=3^{+}\right)$and $\left(n_{b}=3^{-}, n_{b}=\right.$ $\left.2^{+}\right)$increase faster than the energies of $\left(n_{m}=1^{-}, n_{m}=0^{+}\right)$ and $\left(n_{m}=0^{-}, n_{m}=1^{+}\right)$leading to the formation of the double peak shown in the inset.

\section{CONCLUSIONS}

We made use of the continuum approximation to describe the TLG with both, $A B A$ (Bernal) and $A B C$ (rhombohedral), stacking sequences by a $6 \times 6$ Hamiltonian in which we use the Peierls substitution together with the Landau gauge to include an external magnetic field. Using this approach we were able to find analytical expressions for the six-component spinor that we use in order to calculate, within the dipole approximation, the oscillator strength of the transitions between the Landau levels. We also calculated the cyclotron resonance spectrum for neutral and biased layers.

We found that the effect of an applied bias on the transitions between the LL are threefold: reduction of the transition energies, lifting of the degeneracy of the level $n=0$ (twofold degeneracy in $A B C$ TLG and fourfold degeneracy in $A B A$ TLG), and the breaking of the electron-hole symmetry. The first and second effects can be easily observed in the CR spectrum for both types of stacking: reduction of transition energies causes a shift of the peaks and the lifting of the degeneracy appears as extra peaks. Although the asymmetry between electrons and holes has a considerable influence on the OS of the transitions, the difference in the transition energies are rather small, usually smaller than the broadening of $\mathrm{CR}$ peaks, which will make it difficult to see this asymmetry in the CR spectrum, with the exception of the transitions $\left(n_{m}=1^{-}, n_{b}=1^{+}\right)$and $\left(n_{b}=1^{-}, n_{m}=1^{+}\right)$in $A B A$ TLG which occur at very distinguished energies.

It is worth mentioning that in the present work we have neglected the trigonal warping effect caused by the coupling parameters $\gamma_{3}$ and $\gamma_{2}{ }^{28}$ We expect the inclusion of these terms will split the $n=0$ Landau level into three new ones which will cause the appearance of new lines in the cyclotron spectrum due to the additional transitions involving the lowest levels. Nonetheless, this splitting will be very small (i.e., on the $\mathrm{meV}$ level) and for current trilayer graphene devices it is expected to be of no relevance because of the presence of disorder which is at least on the $10 \mathrm{meV}$ level.

\section{ACKNOWLEDGMENTS}

This work was supported by the National Council for the Improvement of Higher education (CAPES), the Brazilian Council for Research (CNPq), the Flemish Science Foundation (FWO-Vl), the bilateral projects between Flanders and Brazil and the CNPq and FWO-Vl, and the ESF-Eurographene project CONGRAN.

\section{APPENDIX A}

The constants appearing in $\Psi_{n}^{A B C}$ are given by

$$
\begin{aligned}
b_{n} \equiv & \frac{1}{\gamma_{1}^{\prime}}\left(\delta_{3}^{n}-\frac{2 n}{l_{B}^{2} \delta_{3}^{n}}\right) \\
c_{n} \equiv & \frac{l_{B}}{2(n+1)}\left(\delta_{2}^{n} b_{n}-\gamma_{1}^{\prime}\right) \\
d_{n} \equiv & \frac{1}{\gamma_{1}^{\prime}}\left(\delta_{2}^{n} c_{n}-\frac{b_{n}}{l_{B}}\right) \\
A_{n} \equiv & \left(\frac{1}{\sqrt{\pi} l_{B} g_{n}}\right)^{1 / 2}, \\
g_{n} \equiv & 2^{n} n !\left(1+b_{n}{ }^{2}\right)+2^{n+1}(n+1) !\left(c_{n}{ }^{2}+d_{n}{ }^{2}\right) \\
& +2^{n+2}(n+2) ! \frac{d_{n}{ }^{2}}{\delta_{1}^{n 2} l_{B}^{2}}+2^{n-1}(n-1) ! \frac{4 n^{2}}{\delta_{3}^{n 2} l_{B}^{2}}
\end{aligned}
$$

where $\gamma_{1}^{\prime}=\gamma_{1} /\left(\hbar v_{F}\right)$. 
The integrals involved in the calculation of the oscillator strength for $A B C$ TLG are given by

$$
\begin{aligned}
& \int \Psi_{A_{1}}^{m *} \bar{x} \Psi_{A_{1}}^{n} d \bar{x}=\sqrt{\pi} 2^{m+1}(m+1) ! \frac{d_{m} d_{n} A_{m} A_{n}}{2}\left\{2(m+2) \delta_{m+2, n+1}+\delta_{m, n+1}\right\}, \\
& \int \Psi_{B_{1}}^{m *} \bar{x} \Psi_{B_{1}}^{n} d \bar{x}=\sqrt{\pi} 2^{m+2}(m+2) ! \frac{d_{m} d_{n} A_{m} A_{n}}{2 \delta_{1}^{m} \delta_{1}^{n} l_{B}^{2}}\left\{2(m+3) \delta_{m+3, n+2}+\delta_{m+1, n+2}\right\}, \\
& \int \Psi_{B_{2}}^{m}{ }^{*} \bar{x} \Psi_{B_{2}}^{n} d \bar{x}=\sqrt{\pi} 2^{m+1}(m+1) ! \frac{c_{m} c_{n} A_{m} A_{n}}{2}\left\{2(m+2) \delta_{m+2, n+1}+\delta_{m, n+1}\right\}, \\
& \int \Psi_{A_{2}}^{m *} \bar{x} \Psi_{A_{2}}^{n} d \bar{x}=\sqrt{\pi} 2^{m} m ! \frac{b_{m} b_{n} A_{m} A_{n}}{2}\left\{2(m+1) \delta_{m+1, n}+\delta_{m-1, n}\right\}, \\
& \int \Psi_{A_{3}}^{m} \bar{x} \Psi_{A_{3}}^{n} d \bar{x}=\sqrt{\pi} 2^{m-1}(m-1) ! \frac{2 m n A_{m} A_{n}}{\delta_{3}^{m} \delta_{3}^{n} l_{B}{ }^{2}}\left\{2 m \delta_{m, n-1}+\delta_{m-2, n-1}\right\}, \\
& \int \Psi_{B_{3}}^{m *} \bar{x} \Psi_{B_{3}}^{n} d \bar{x}=\sqrt{\pi} 2^{m} m ! \frac{A_{m} A_{n}}{2}\left\{2(m+1) \delta_{m+1, n}+\delta_{m-1, n}\right\},
\end{aligned}
$$

where $m$ and $n$ are Landau level indices for the different eigenstates. The selection rule is as usual $|m-n|=1$. The result of Eq. (A2e) is for $m, n>0$; otherwise, this integral is zero.

\section{APPENDIX B}

The constants appearing in $\Psi_{n}^{A B A}$ are given by

$$
\begin{aligned}
b_{1(3)}^{n} & \equiv \frac{1}{\gamma_{1}^{\prime}}\left(\frac{2 n}{l_{B}}-l_{B} \delta_{1(3)}^{n}{ }^{2}\right), \\
B_{n} & \equiv\left(\frac{1}{\sqrt{\pi} l_{B} g_{n}}\right)^{1 / 2}, \\
g_{n} & \equiv 2^{n-2}(n-2) ! \frac{4(n-1)^{2} b_{1}^{n 2}}{\delta_{2}^{n 2} l_{B}^{2}}+2^{n-1}(n-1) !+2^{n-1}(n-1) !\left[l_{B}^{2} \delta_{1}^{n 2}+b_{1}^{n 2}+l_{B}^{2} \delta_{3}^{n 2}\left(\frac{b_{1}^{n}}{b_{3}^{n}}\right)^{2}\right]+2^{n} n !\left[1+\left(\frac{b_{1}^{n}}{b_{3}^{n}}\right)^{2}\right] .
\end{aligned}
$$

The integrals needed to calculate the oscillator strength of the transitions are the following:

$$
\begin{aligned}
& \int \Psi_{A_{1}}^{m *} \bar{x} \Psi_{A_{1}}^{n} d \bar{x}=\sqrt{\pi} 2^{m-1}(m-1) ! \frac{\delta_{1}^{m} \delta_{1}^{n} B_{m} B_{n}}{2}\left\{2 m \delta_{m, n-1}+\delta_{m-2, n-1}\right\}, \\
& \int \Psi_{B_{1}}^{m *} \bar{x} \Psi_{B_{1}}^{n} d \bar{x}=\sqrt{\pi} 2^{m} m ! \frac{B_{m} B_{n}}{2}\left\{2(m+1) \delta_{m+1, n}+\delta_{m-1, n}\right\}, \\
& \int \Psi_{B_{2}}^{m *} \bar{x} \Psi_{B_{2}}^{n} d \bar{x}=\sqrt{\pi} 2^{m-1}(m-1) ! \frac{b_{1}^{m} b_{1}^{n} B_{m} B_{n}}{2}\left\{2 m \delta_{m, n-1}+\delta_{m-2, n-1}\right\}, \\
& \int \Psi_{A_{2}}^{m *} \bar{x} \Psi_{A_{2}}^{n} d \bar{x}=\sqrt{\pi} 2^{m-2}(m-2) ! \frac{2 b_{1}^{m} b_{1}^{n} B_{m} B_{n}(m-1)(n-1)}{l_{B}^{2} \delta_{2}^{m} \delta_{2}^{n}}\left\{2(m-1) \delta_{m-1, n-2}+\delta_{m-3, n-2}\right\}, \\
& \int \Psi_{A_{3}}^{m *} \bar{x} \Psi_{A_{3}}^{n} d \bar{x}=\sqrt{\pi} 2^{m-1}(m-1) ! \frac{l_{B}{ }^{2} \delta_{3}^{m} \delta_{3}^{n} b_{1}^{m} b_{1}^{n} B_{m} B_{n}}{2 b_{3}^{m} b_{3}^{n}}\left\{2 m \delta_{m, n-1}+\delta_{m-2, n-1}\right\}, \\
& \int \Psi_{B_{3}}^{m *} \bar{x} \Psi_{B_{3}}^{n} d \bar{x}=\sqrt{\pi} 2^{m} m ! \frac{b_{1}^{m} b_{1}^{n} B_{m} B_{n}}{2 b_{3}^{m} b_{3}^{n}}\left\{2(m+1) \delta_{m+1, n}+\delta_{m-1, n}\right\} .
\end{aligned}
$$

Equations (B2a), (B2c), and (B2e) are valid for $m, n>0$, while Eq. (B2d) is valid only for $m, n>1$; otherwise, these integrals are zero.

\footnotetext{
${ }^{1}$ A. H. Castro Neto, F. Guinea, N. M. R. Peres, K. S. Novoselov, and A. Geim, Rev. Mod. Phys. 81, 109 (2009).

${ }^{2}$ M. L. Sadowski, G. Martinez, M. Potemski, C. Berger, and W. A. de Heer, Phys. Rev. Lett. 97, 266405 (2006).

${ }^{3}$ Z. Jiang, E. A. Henriksen, L. C. Tung, Y.-J. Wang, M. E. Schwartz, M. Y. Han, P. Kim, and H. L. Stormer, Phys. Rev. Lett. 98, 197403 (2007).
}

${ }^{4}$ V. P. Gusynin, S. G. Sharapov, and J. P. Carbotte, Phys. Rev. Lett. 98, 157402 (2007).

${ }^{5}$ R. S. Deacon, K.-C. Chuang, R. J. Nicholas, K. S. Novoselov, and A. K. Geim, Phys. Rev. B 76, 081406(R) (2007).

${ }^{6}$ V. P. Gusynin and S. G. Sharapov, Phys. Rev. Lett. 95, 146801 (2005). 
${ }^{7}$ K. S. Novoselov, A. K. Geim, S. V. Morozov, D. Jiang, M. I. Katsnelson, I. V. Grigorieva, S. V. Dubonos, and A. A. Firsov, Nature (London) 438, 197 (2005).

${ }^{8}$ Y. Zhang, Y. W. Tan, H. L. Stormer, and P. Kim, Nature (London) 438, 201 (2005).

${ }^{9}$ T. Ohta, A. Bostwick, T. Seyller, K. Horn, and E. Rotenberg, Science 313, 951 (2006).

${ }^{10}$ H. Min, B. Sahu, S. K. Banerjee, and A. H. MacDonald, Phys. Rev. B 75, 155115 (2007).

${ }^{11}$ E. V. Castro, K. S. Novoselov, S. V. Morozov, N. M. R. Peres, J. M. B. Lopes dos Santos, J. Nilsson, F. Guinea, A. K. Geim, and A. H. Castro Neto, Phys. Rev. Lett. 99, 216802 (2007).

${ }^{12}$ J. M. Pereira, Jr., F. M. Peeters, and P. Vasilopoulos, Phys. Rev. B 76, 115419 (2007).

${ }^{13}$ E. A. Henriksen, Z. Jiang, L.-C. Tung, M. E. Schwartz, M. Takita, Y.-J. Wang, P. Kim, and H. L. Stormer, Phys. Rev. Lett. 100, 087403 (2008).

${ }^{14}$ K. S. Novoselov, E. McCann, S. V. Morozov, V. I. Fal'ko, M. I. Katsnelson, U. Zeitler, D. Jiang, F. Schedin, and A. K. Geim, Nature Phys. 2, 177 (2006).

${ }^{15}$ A. A. Avetisyan, B. Partoens, and F. M. Peeters, Phys. Rev. B 79, 035421 (2009).

${ }^{16}$ A. A. Avetisyan, B. Partoens, and F. M. Peeters, Phys. Rev. B 81, 115432 (2010).
${ }^{17}$ C. H. Lui, Z. Li, K. F. Mak, E. Cappelluti, and T. F. Heinz, Nature Phys. 7, 944 (2011).

${ }^{18}$ W. Bao, L. Jing, J. Velasco, Jr., Y. Lee, G. Liu, D. Tran, B. Standley, M. Aykol, S. B. Cronin, D. Smirnov, M. Koshino, E. McCann, M. Bockrath, and C. N. Lau, Nature Phys. 7, 948 (2011).

${ }^{19}$ S. H. Jhang, M. F. Craciun, S. Schmidmeier, S. Tokumitsu, S. Russo, M. Yamamoto, Y. Skourski, J. Wosnitza, S. Tarucha, J. Eroms, and C. Strunk, Phys. Rev. B 84, 161408(R) (2011).

${ }^{20}$ S. Yuan, R. Roldán, and M. I. Katsnelson, Phys. Rev. B 84, 125455 (2011).

${ }^{21}$ S. H. R. Sena, J. M. Pereira, Jr., F. M. Peeters, and G. A. Farias, Phys. Rev. B 84, 205448 (2011).

${ }^{22}$ T. Taychatanapat, K. Watanabe, T. Taniguchi, and P. Jarillo-Herrero, Nature Phys. 7, 621 (2011).

${ }^{23}$ A. Kumar, W. Escoffier, J. M. Poumirol, C. Faugeras, D. P. Arovas, M. M. Fogler, F. Guinea, S. Roche, M. Goiran, and B. Raquet, Phys. Rev. Lett. 107, 126806 (2011).

${ }^{24}$ F. Guinea, A. H. Castro Neto, and N. M. R. Peres, Phys. Rev. B 73, 245426 (2006).

${ }^{25}$ M. Koshino and E. McCann, Phys. Rev. B 83, 165443 (2011).

${ }^{26}$ B. Partoens and F. M. Peeters, Phys. Rev. B 75, 193402 (2007).

${ }^{27}$ H. Min and A. H. MacDonald, Phys. Rev. B 77, 155416 (2008).

${ }^{28}$ M. Koshino and E. McCann, Phys. Rev. B 80, 165409 (2009). 